\title{
THE EFFECT OF TEACHING "CHAOS THEORY AND FRACTAL GEOMETRY" ON GEOMETRIC REASONING SKILLS OF SECONDARY STUDENTS
}

\author{
Reda Abu-Elwan, PhD \\ Sultan Qaboos University, Muscat, Oman
}

\begin{abstract}
Chaos theory and fractal geometry have begun to appear as an important issue in secondary school mathematics. Chaos theory is the qualitative study of unstable periods in deterministic nonlinear dynamical systems, chaos theory looks at how things evolve. Fractal geometry is a subject that has established connections with many areas of mathematics (including number theory, probability theory and dynamical systems). Fractal geometry, together with the broader fields of nonlinear dynamics and complexity, represented a large segment of modern science at the end of the $20^{\text {th }}$ century; this paper investigates the concepts of chaos theory and fractal geometry as a conceptual transformation at secondary school level. This paper reports a study of the effects of teaching chaos theory and fractal geometry on geometric reasoning skills in geometry. Thirty of the tenth grade students of basic education participated in an experimental group, which was involved in working with chaos theory and fractal geometry activities, pre-treatment measures the geometric Reasoning skills. Teaching fractal geometry properties and examples were focused in the teaching activities. At the end of the teaching measures geometric reasoning skills were again obtained. Since the study was an exploration, the effectiveness of teaching chaos theory and fractal geometry, the exploratory data collected by the researcher was also considered to be an important part of the study.
\end{abstract}

\section{Keywords:}

Chaos theory and fractal geometry; Geometric reasoning skills; Iteration geometry

\section{Council for Innovative Research}

Peer Review Research Publishing System

\section{Journal: INTERNATIONAL JOURNAL OF RESEARCH IN EDUCATION METHODOLOGY}

Vol. 6, No.2

www.ijrem.com, ijremeditor@gmail.com 


\section{Introduction}

Geometry is integrated in an inclusive mathematics curriculum in Oman secondary stage. Clements and Battista (1992) have claimed that school geometry refers almost universally to Euclidian geometry, even though there are numerous approaches to the study of a topic, such as synthetic, analytical, transformational, and vector- not to mention nonEuclidian approaches. The National Council of Supervisors of Mathematics(NCSM) identified geometry as one of the ten basic skill areas of mathematics, in their 1978 position statement NCSM stated, "students should learn the geometric concepts they will need to function effectively in the three-dimensional world" (p. 149)

Secondary geometry builds on middle and elementary school geometry, which traditionally has emphasized measurement and informal development of the basic concepts required in geometry at secondary school level, fractal geometry provides an accessible, interesting setting not only for mathematical research, but also for mathematics education, and there have been many curricular initiatives in this area. In addition to its impact on middle and secondary school mathematics, fractal geometry has influenced undergraduate mathematics, and has been the source for many undergraduate research efforts. The difference between chaos and fractals is the fact that chaos depends on the dynamics more than the geometric properties of the system, while for fractals the opposite is true (Stewart, 1998\& 216). In this study, I have investigated the effect of teaching chaos theory and fractal geometry as an applied part of geometry and its geometric properties upon geometric reasoning skills of the secondary students, this experimental study took place during the semester of autumn of 2013.

\section{Theoretical Background}

Mathematics is an experimental science, a discipline that is still evolving and making new discoveries. Reform in mathematics mirrors the reforms proposed by cognitive research. In the year 2000 NCTM principles and standards, reflect a very different attitude toward the teaching of mathematics from the traditional algorithmic approach by placing an emphasis on doing mathematics as well as well knowing mathematics. Reasoning is central to mathematics as a discipline (Steen, 1997) and underpins mathematical learning (Russell, 1999). As NCTM (2000) emphasizes, the ability to reason is essential to mathematical understanding, and should be a primary goal in mathematics education by developing ideas, exploring phenomena, justifying results, and using mathematical conjectures in all content areas. Chaos theory and fractal geometry are the result of this change and fall within the boundaries of discrete mathematics (Fareell, 1998). Chaos theory and fractal geometry provide an opportunity for students to understand that mathematics itself is evolving and changing, it provides an opportunity for students to investigate and describe the relationship between geometry and algebra and further the understanding of dynamical system and mathematics. Fractals demonstrate a repetitive scaling that makes them identical on all scales, whereas chaos introduces a seemingly random aspect to the patterning.

\section{Chaos theory}

Chaos is a topic that has developed through the study of dynamical systems and has connection with fractal geometry, fractals can be thought of as the images of chaos. Geometrically, they exist in between our familiar dimensions. Infinitely complex, their patterns are self-similar across different scales. Chaos Theory deals with nonlinear things that are effectively impossible to predict or control, like turbulence, the stock market, weather forecasters use patterns in weather to predict temperature, tornadoes, and hurricanes. Some aspects of weather forecasting use chaos theory. Many natural objects exhibit fractal properties, including landscapes, clouds, trees, organs, rivers etc, and many of the systems in which we live exhibit complex, chaotic behavior. Chaos involves systems or equations that are extremely sensitive to initial conditions and that preclude a prediction of long term behavior as a result of the inability to perfectly define the initial conditions such as the weather. Chaotic systems often appear random and unpredictable but they are often defined by simple equations and demonstrate intermittent periodic behavior. Chaotic systems are known as non-linear equations meaning that a small change in the initial parameters does not result in a small change in the outcome, but instead is widely divergent and results in unpredictable outcomes. Recognizing the chaotic, fractal natural of our world can give us new insight, power, and wisdom. According to Kellert (1993), chaos theory is the qualitative study of unstable a periodic behavior in deterministic nonlinear dynamical systems. A dynamical system is one that changes over time, so chaos theory looks at how things evolve. Clapham describes chaos as a situation in which a fully deterministic dynamical process can appear to be random and unpredictable due to the sensitive dependence of the process on its starting values and the wide range of qualitatively different behaviors available to the process. This sensitive dependence is often called the butterfly effect (Clapham, 1996;35). The systems studied are deterministic, that is, the state of the system at any stage depends on the state at the previous stage in a way that can be completely specified using simple mathematical operations on exact numbers (Bedford, 1998; 276). Chaos is defined in systems that are incredibly sensitive to initial conditions. Linear systems can be represented by the equation, $x \rightarrow a x+b$. Unlike linear equations, in which changes in initial conditions have a proportional effect, in a chaotic system very small changes in initial conditions can, and often do result, in totally divergent outcomes.

The main tool for studying a dynamical system is the sequence, called an orbit in chaos theory. The sequence is generated by iterating a function, $f$, whose initial argument, $x$, is called a seed. The three defining characteristics of deterministic chaos according to Devaney (1992) are:

Sensitivity to initial conditions: Arbitrarily close to every seed is another whose orbit moves far. Mixing of the domain: if we specify any two subintervals of the domain, we can always find a seed in the first interval whose orbit gets into the second. 
Density of periodic points: any point of the domain is arbitrarily close to a point whose orbit ends up in a repetitive cycle. I presented chaotic behavior of a point for secondary students by asking them to choose a point xo, find where the tangent at $\frac{1}{2}\left(\mathrm{x}-\frac{1}{\mathrm{x}}\right)$ meets the $\mathrm{x}$-axis, and repeat the process over and over again. What do we think will happen? To understand how chaos work I asked them to do the following:

Sketch a graph of $y=x^{2}+1$. Starting anywhere you like, carry out Newton's method construction a few times on your graph. What would happen if, at some stage in the iteration, you reached a very positive number, or a very small negative number?

Show that Newton's method applied to the equation $x^{2}+1=0$ is equivalent to iterating the functionf $(x)=\frac{1}{2}\left(x-\frac{1}{x}\right)$.

Choose any number as starting point, and carry out the iteration at least 10 times. Do you see any pattern in the sequence?

Choose another starting point very close to the first one, and iterate the same number of times. Compare the last few numbers in the two sequences. What do you notice?

The sequence has no detectable pattern, and very small differences in the starting point can result in large differences further along the sequence. If a sequence like this were used to model a real world system, it would be impossible to predict future outcomes.

One of the interesting approaches to the introduction of chaos for secondary students is the chaos game approach to fractals suggested by (Devancy, 2004) which provides teachers with an opportunity to help students comprehend the geometry of affine transformations. The classical chaos game and other related games are described in Deviancy's article. Crilly (1991) described the principal features of chaos, as it is the simple deterministic systems can generate what appears to be random behavior. Chaos can be observed in basic mathematical systems (Crilly et al., 1991), 195).

\section{Fractal geometry}

Fractal geometry has been explored in classrooms from kindergarten through graduate school for more than twenty-five years. Fractal geometry is an irregular geometric object with an infinite nesting of structure at all scales. Fractals are repetitive patterns that display characteristics of scaling and self similarity. Fractal geometry has become a topic of widespread interest in recent years. It serves as a powerful illustration of some of the qualities of mathematical inquiry that have been underdressed in school mathematics (Simmt \& Davies, 1999, 103). The study of fractal geometry is appropriate in high school for many reasons: students have the opportunity to investigate traditional mathematics topics from a new approach, to make connections both within mathematics and between mathematics and natural and human worlds, and to explore mathematics in non-analytic ways (Lornell\&Wesberg, 1999, 265). Fractal geometry allows students to explore mathematical concepts by drawing pictures of constructive iteration of classical fractals.

The word Fractal comes from the Latin verb franger which means to break (Gleik, 1987). This verb refers to a quality often characterizing natural objects; they look fragmented, irregular, broken, complex (Lornell \& Wesberg, 1999). There are two main properties for fractal geometry: first is self-similarity, with this attribute, smaller sections of the object (fractal) are replicas of the whole object. Strict self-similarity refers to a characteristic of a form exhibited when a substructure resembles a superstructure in the same form. Self-similarity can be found in many fractals. Students can see geometric patterns and they begin to able to describe the relationship of these patterns (Bringer\& Ury, 2002). Second is the fractal dimension:

Peitgen (1992) describe the foundation of fractal dimension; given a self-similar structure, there is a relation between the reduction factors and the number of pieces a into the structure can be divided, and that is

$$
\mathrm{a}=\frac{\log \mathrm{a}}{\log \frac{1}{\mathrm{~s}}} \text { Or } \mathrm{D}=\frac{\log \mathrm{a}}{\log \frac{1}{\mathrm{~s}}}
$$

D is called the self-similarity dimension, for the line, the square and the cube we obtain the expected self-similarity dimensions one, two, and three (Peitgen, 1992, 232-233). Fractal scaling means that the same level of detail exists at all levels. Scaling is the property of being the same on all scales

Fractals can be divided into two categories (Vacc, 1999): natural fractals and mathematically structured fractals. This study concentrates on the mathematically structured fractals and using some examples of natural fractals in the everyday environment of students to be an attractive approach for studying fractal geometry and chaos theory. Teaching fractal concepts requires more activities so that students can be involved in drawing, reasoning, and mathematical thinking. In the procedure of learning fractal geometry, observation of the construction of fractal images is the most important part as much as understanding mathematical ideas behind them. Students will understand mathematical concepts better if they could see the construction procedure of the fractal figures step by step (Yi, 2004).

\section{Geometric Reasoning Skills}

Reasoning in the classroom is affected by teacher expectations, the classroom discourse, and opportunities to make sense of mathematics through different types of reasoning and more general conditions such as a supportive climate (Diezmann, et al., 2002). Developing student's geometric reasoning skills is one of the major objectives of geometry teaching in secondary mathematics. 
As fractal geometry evolves to encompass the understanding of diverse natural phenomena, it is important to be clear about what is meant by the geometrical reasoning necessary to understand the components of natural fractal images and how such reasoning develops. Although geometry consists of the geometric figures of zero, one, two, and three dimensions and relationships among them, fractal geometry present a new vision of geometric figures of fractal dimension that lies between two and three dimensions. Students study properties and relationships having to do with size, shape, location, direction and orientation of these figures. As Davis $(2004 ; 192)$ has pointed out: The value of fractal geometry to studies of education is just beginning to be demonstrated. For example, in terms of description, the notion of scale independence and self-similarity are useful for making sense of the leveled and embedded natures of individuals, social collectives, bodies of knowledge, cultures and societies, O'Daffer and Thorquist (1993; 43) have pointed out that mathematical reasoning is part of mathematical thinking that involves forming generalizations and drawing valid conclusions about ideas and how they are related. Mathematical reasoning can be viewed as a dynamic activity that includes a variety of modes of thinking. It is an integral component of mathematical thinking (Pressini \& Webb, 1999; 157).

Teaching fractal geometry of secondary students presents a strong geometry-learning environment to develop geometric reasoning skills. Inductive reasoning would be when we are given a geometric pattern and we need to come up with the rule for the pattern. There is a clear example for inductive reasoning skills in fractal shapes. Figure 1 show that Example:

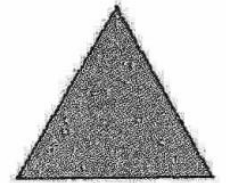

Stage 0



Stage 1

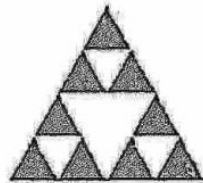

Stage 2

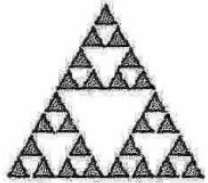

Stage 3



Stage 4

\title{
Figure 1: The Sierpinski triangle
}

\author{
Students can explore numerical relationship and generalize as it showed \\ below
}

\begin{tabular}{|c|c|c|c|c|c|c|}
\hline Stage & 0 & 1 & 2 & 3 & 4 & $n$ \\
\hline N. of shaded triangles & 1 & 3 & 9 & 27 & 81 & $3^{n}$ \\
\hline N. of holes & 0 & 1 & 4 & 13 & 40 & $3^{n}-1$ \\
\hline Areas of shaded pieces & 1 & & $\frac{9}{16}$ & $\frac{27}{64}$ & $\frac{81}{256}$ & $\left(\frac{1}{1}\right)^{n}$ \\
\hline $\begin{array}{c}\text { Perimeter around shaded } \\
\text { pieces }\end{array}$ & 1 & $\frac{3}{2}$ & $\frac{9}{4}$ & $\frac{27}{8}$ & $\frac{81}{16}$ & $\left(\frac{3}{2}\right)^{n}$ \\
\hline
\end{tabular}

The Royal Society/ Joint Mathematical Council (2001) report on the teaching and learning of geometry for pupils aged 11 19 makes a number of recommendations about suitable approaches to the teaching of deductive reasoning in geometry. The report suggests that (p. 5):

Geometric situations should be chosen, as far as possible, to be useful, interesting and/or surprising to pupils, and the level of sophistication expected in the logical argument will depend upon the age and attainment of the pupils concerned, and the proof produced might equally be called an explanation, or justification or reason for the result.

Several studies have described geometric reasoning skills; the Van Hieles (1986) have described a progression in geometry curriculum. The levels are:

$>$ Identification or production of a shape by visual recognition (e.g. recognizing or drawing a rhombus);

$>$ Awareness of properties of classes of shape (observing that a rhombus has four equal sides but no right angles);

$>$ Beginning to clarify definitions or relations between different shapes and properties and to make some logical connections;

$>$ Developing deductive reasoning, deriving new theorems from one or more axioms or theorems accepted as true; 
$>$ Appreciation of the abstract structure of an axiomatic system, with axioms as an initial set of statements accepts as true, and a network of theorems, which can be derived from these.

Brown, Jones, and Taylor (2003) have described deductive geometrical reasoning to include Theorems, is an important aim of a mathematics curriculum. However, deductive geometrical reasoning can be more widely interpreted to also include:

$>$ Deriving a specific value of a variable (e.g. the size of an angle) using both known theorems and known prosperities of shapes.

$>$ Deducing a specific result in relation to a figure with given properties which does not have the generality or status of a theorem (e.g. proving that two sides of a quadrilateral with a particular set of properties are equal). This type of problem used to be known as a rider.

$>$ Considering an alternative definition of geometrical shapes, deciding which of these are necessary, sufficient and minimal, and becoming familiar with the differences between the meanings of these terms. (p.6)

Duval (1998) pointed out that geometrical reasoning involves three kinds of cognitive processes:

> Visualization processes, for example the visual representation of a geometrical statement, or the heuristic exploration of a complex geometrical situation.

$>$ Construction processes (using tools)

$>$ Reasoning processes- particularly discursive process for the extension of knowledge, for explanation, and for proof. ( $p$. 38)

Fractal geometry is a natural place for the development of students reasoning skills; it offers ways to describe physical environments and natural geometric figures.

\section{Objective of the study}

The purposes of this study were:

1. To develop teaching activities in chaos theory and fractal geometry for secondary students.

2. To identify the effectiveness of teaching the suggested activities on developing secondary students geometric reasoning skills.

\section{Research Questions}

The following questions have been identified as the focus for this study:

1. What is the effect of teaching chaos theory and fractal geometry on the achievement of the tenth grade students?

2. What is the effect of teaching chaos theory and fractal geometry on developing the tenth grade students geometric reasoning skills?

\section{Hypotheses of the study}

The study included two hypotheses, which are listed below:

1. There are statistically significant differences $(p<0.05)$ between students mean scores in the achievement pre-test and their mean scores in the same achievement post-test in favor of the students mean scores of the post-test.

2. There are no statistically significant differences $(p<0.05)$ between students mean scores in Geometric reasoning skills pre-test and their mean scores in the same post-test in favor of the students mean scores of the post-test.

Overview of Chaos Theory and Fractal geometry teaching activities:

The objectives of chaos theory and fractal geometry teaching activities for secondary students were to explore the phenomena of chaos, which refers to fractals, and to develop fractal geometry concepts based on the iteration geometry. These activities center primarily around fractals with a small emphasis on chaos theory, the study of chaos and fractals both deal with the structure of irregularity

The content of teaching activities includes the following:

\section{First: Three specific projects (groups activate):}

1. The chaos game: the goal of the Chaos game is to improve students geometric thinking, it is connected the role of the chaos game in construction fractals.

2. Construction of the Siepinski Tetrahedron.

3. The generation pattern of Pascal's Triangle: Students will use iterative geometric constructions to create the Sierpinski triangle as well as a variation of it. On closer inspection, students will find that the numerical patterns within these triangles demonstrate an amazing link between algebra and geometry.

The groups' activities have been designed to practice the wonderful side of the chaos and fractal geometry by students; they apply the fractal properties to construct the Seierpinski tetrahedron and generation pattern of Pascal's triangle. Also students discovered the relationship between chaos and fractal through the chaos game. 


\section{Second: Teaching Fractal geometry (individual worksheets activities):}

1. Spirolateral: it is a geometric design generated from number sequences through iterative procedures, it connect to a wide variety of other topics and have obvious applications when discussing symmetry, transformations, and coordinate geometry.

2. The Sierpinski triangle

3. Fractal curves: The Coach curve, The Hat curve.

4. Cantor set

5. Fractal Cards

The design of the activities of the second part was based on educational tasks,so that each student could practice reasoning skills, which appear in each task. In addition, they understand the concepts of fractal geometry. Resources of the suggested activities were from: two books ( Sobel and Maletsky, 1999 \& Malestsky; perciante; and Yunker, 1992), and internet website such as:

http://math.bu.edu/DYSYS/applets/chaos-game more.html

$\underline{\text { http://metalinks.metaculture.net/science/fractal/learning/default.asp }}$

$\underline{\text { http://home.inreach.com/kfarrell/course.outline.html }}$

Some of the mathematics educators (three teachers and three of my colleagues) reviewed all the teaching activities, they provided feedback and comments on the content of activities and I adjusted the content accordingly.

\section{Methods}

Participants

The participants in this study were students of the tenth grade in a secondary school in Muscat area (grades tenth, eleventh, and twelfth are secondary grades in Omani educational system). The thirty participants were all female and had a secondary mathematical background, which is required to understand the topics of chaos theory and fractal geometry.

\section{Instruments:}

In order to gather data, two instruments were used in this study:

\section{First: An Achievement Test in chaos theory and fractal theory}

\section{(ATCTFG):}

The main purpose of using the achievement test was to measure students' achievement on the content of chaos theory and fractal geometry. ATCTFG consists of six questions, each question requiring writing all solving steps. The question dealt with cognitive aspects of chaos theory and fractal geometry, these aspects were chosen from the work of (Benson et al., 1993\& and Barnes, 1993), The selection, and adoption of some of the question, was done in consultation with four colleagues in math department in college of Science and math education in college of Education at SQU, also it was refereed by three experts who are working in the field of fractal geometry in USA. Resources of test Questions were chosen to assess chaos theory concepts (Qs. \#1 and \#3), fractal geometry skills (Qs. \# 2, \#4, \#5, and \#6). The initial results of the test for a group of 25 students (chosen from another school and has not studied fractal geometry) yielded Cronbach alpha reliability coefficient of 0.67 . (Appendix1).

\section{Second: Geometric Reasoning Skills Test}

\section{(GRST):}

The research study planned to evaluate the effectiveness of teaching chaos theory and fractal geometry on geometric reasoning skills for secondary students, the researcher designed Geometric Reasoning skills Test (GRST) for the purpose of the study. Several steps have been done to develop the GRST; determined its objectives, determined the geometric content, choose the effective questions, then measuring the validity and stability of the test. Referees commented and suggested changes on the second part of the test, the researcher did the recommended comment. GRST consists of two parts: first part consists of three questions, each one of them asked students to draw and filling the missing information based on the presented fractal figures, second part consists of four questions, these questions asked students to choose the right answer based on his skills of geometric reasoning content of the question, The suggested time for the test by the referees was 120 minutes. The total marks of the test estimated as 20 marks: 4 marks for each question of the first part, and 2 marks for each question of the second part, A Cronbach alpha reliability coefficient of the test was 0.63. (Appendix 2)

\section{The Treatment in the Experimental Group}

This is a quasi-experimental study of one sample group, in which topics of chaos theory and fractal geometry activities in addition to school geometry has presented for the experimental group. Because of the new content of chaos and fractal geometry, which is not presented to all students of the same grade, it was suitable for only one sample experimental 
group, Students were taught the additional geometric activities (chaos theory and fractal geometry) in separate periods (each period-lasted 40 minutes) a week in a five weeks at the same pace in the first tem of the 2013 academic year.

The geometric activities in fractal geometry designed in continuous teaching tasks, each task contained an objective with activities that should be done by students and an evaluation about the task content.

Tasks were presented to students with a clear instruction by the teacher. Since the teacher had never taught fractal geometry before but was willing to teach it in the classroom, I assumed most of the responsibility for both the tasks planning and the carrying out of the experience. During the first five minutes, warm up activities were used to make the students ready to be involved in the content tasks. Additional internet websites resources were used to develop understanding of fractal properties, for examples:

www.yale.edu/fractals

www.fractalfoundation.org

The students worked in groups of 4-6 students. They were active by taking roles, imagining, discussing, exploring, deciding, justifying, expressing ideas, and communicating during the lesson. The two instruments: ATCTFG and GRST have presented to students of the experimental group before teaching the suggested fractal geometry activities, and after teaching.

\section{Results and Discussion:}

This study examined the effectiveness that studying chaos theory and fractal geometry activities had on students' achievement and their geometric reasoning skills. Obtained data were analyzed by using SPSS v.12.0 statistical computer software. To answer the research questions, t-test were conducted in order to compare the mean scores of the experimental group on pre and post achievement test. Table 1 presents the means and standard deviations for the achievement on which the pre and post of achievement tests differed significantly.

Table 1: Means, Standard Deviations, and Value of T-Test for Achievement Test

\begin{tabular}{|l|l|l|l|l|l|l|}
\hline & $\mathrm{N}$ & Means & SD & T-value & df & $\mathrm{P}<$ \\
\hline $\begin{array}{l}\text { Pre-achieve. } \\
\text { test }\end{array}$ & 30 & 13.50 & 8.62 & & & \\
\hline $\begin{array}{l}\text { Post- } \\
\text { achieve. } \\
\text { test }\end{array}$ & 30 & 37.50 & 10.73 & 17.32 & 29 & 0.000 \\
\hline
\end{tabular}

- Stands for $p>0.05$

The independent t-test results of achievement test showed that student's performances for post achievement test has improved in which posttest mean score was 37.5, and S.D. was 10.73 compared by students performance in pre achievement test in which mean score was 13.5, and S.D. was 8.62. Table 1 shows that there is a significant statistical differences at the level of $p<0.05$ for $t$ value of 17.32 between students performances of the achievement test for the favorite of the posttest. These results could indicate that participants demonstrated improved performances in the achievement of chaos theory and fractal geometry activities which were presented to them in this study. The first hypothesis has been accepted as it is showed in results in table 1.

To identify the effect size of statistical significance of $t$ value, the equation of the effect size (Fam, 1997; 69) used:

$\mu^{2}=0.91$ and when comparing this value with $\mu^{2}$ value on standards table of the effect size, it is clear that the effect size is large. This result means that students can achieve concepts and skills of fractal geometry and chaos theory. Students' performances in the achievement test showed that teaching chaos theory in its suitable levels, the selected fractal geometry activities are effective, and we may integrate these activities in secondary mathematics curriculum. This result coincides with Al-Shahat 's results of her fractal geometry program for secondary students (Al-Shahat, 2005).

To determine the effectiveness of teaching chaos theory and fractal geometry on geometric reasoning skills of the experimental group, t-tests were performed to find out if statistically significant differences existed between students performances on pre and posttest of GRST. Table 2 presents the means and standard deviations for the GRST on which the pre and post of achievement tests differed significantly. 
Table 2: Means, Standard Deviations, and Value of T-test for GRST

\begin{tabular}{|l|l|l|l|l|l|l|}
\hline & $\mathrm{N}$ & Means & SD & T-value & df & $\mathrm{P}<$ \\
\hline Pre-GRST & 30 & 19.83 & 6.76 & 21.07 & 29 & 0.000 \\
\hline $\begin{array}{l}\text { Post- } \\
\text { GRST }\end{array}$ & 30 & 43.16 & 3.6 & & & \\
\hline
\end{tabular}

The independent t-test result of GRST showed that students performances for post test of GRST had improved in which post test mean score was 43.16, and S.D. was3.6 compared by students performance in pre test of GRST in which mean score was 19.83, and S.D. was 6.76. Table 2 shows that there is a statistical significant difference at the level of $p<0.05$ for $t$ value of 21.07 between students performances of the GRST for the favorite of the posttest. These results appear to indicate that participants demonstrated improved performances in pre-test of GRST after the studying of chaos theory and fractal geometry presented for them in this study. Thus, the second hypothesis has been rejected as it is showed in results of table 2 .

To identify the effect size of statistical significance of $t$ value, $n^{2}=0.93$, and comparing this value with $n^{2}$ value on standards table of the effect size, it is clear that the effect size is large. This result means that teaching concepts and skills of fractal geometry and chaos theory can improve geometric reasoning skills of secondary students and it is important to consider that topics in secondary mathematics curricula. The statistical analyses conducted for this study indicate that studying chaos theory and fractal geometry produces significant differences in both achievement and geometric reasoning skills. This result is coinciding with results of (Camp, 1995; Lornell, 1999). Geometric reasoning skills may be developed for secondary students when they learn Euclid geometry, students of the experimental group showed high performance of skills need to solve the pre test of GRST, that means fractal geometry is a rich subject in which geometric reasoning skills may apply.

\section{References}

1. Al-Shahat, Amal (2005). A proposed program in Fractal Geometry by Using the Computer for the Secondary Stage Gifted Students, Unpublished PhD, Ain-Shams University, Cairo.

2. Bedford, Crayton W. (1998). The case for Chaos. The Mathematics Teacher, 91(4), April, 276-281.

3. Brigner, L.; Ury P. (2002). An Adventure in Fractal Geometry; Self= Similarity, Number Pattern and Chaos, Connect: A magazine of Teachers innovations In k-8 Science and Math, 15(5), May/June.

4. Brown, M.; Jones, K. and Taylor, R. (2003). Developing Geometrical reasoning in the Secondary School: Outcomes of trailing teaching activities in classroom, A Report from the Southampton/Hampshire Group to QCA, Nov., QCA.

5. Camp, D. (1995). Fractal Geometry in the High School Classroom, International Reviews on Mathematical Education, V. 27, N. 5, 265-274,

6. Clapham, C. (1996). Concise Dictionary of Mathematics. Oxford University press, New York

7. Clements, D.H., Battista, M.T. (1992). Geometry and spatial understanding. In Dougles A. Grouws (Eds.), Handbook of Research in Mathematics Teaching and Learning, McMillan pub, Co, New York.

8. Crilly, A.J.; Earnshaw, R.A.; and Jones, H. (1991). Fractals and Chaos. New York, Springer-Varlag.

9. Davis, B. (2004). Inventions and Teaching: A Genealgy. Mahwah, NJ: Lawrence Elbaum.

10. Diezmann, C., Waters, j. and English, L. (2002). Teacher Behaviors that influence young Children's Reasoning. In Cockburn, A. and Nardi, E.(Edts.). proceedings of the $26^{\text {th }}$ Conference of the international Group for the Psychology of Mathematics Education, V. 1 (pp. 289-296), Norwich, UK.

11. Duval, R.(1998). Geometry from a Cognitive Point of View. In C Mammana and Villani (Eds), perspectives on the teaching of geometry for the $21^{\text {st }}$ century: an ICMI study. Dordrecht: Kluwer.

12. Devaney, Robert L. (1992). A First Course in chaotic Dynamical Systems: theory and experiment. Reading, Mass: Addison-Wesley Pub.Co.

13. Devaney, Robert L. (2004). Fractal Patterns and Chaos Games, Mathematics Teacher, .98 (4), Nov., $228-223$.

14. Fam, M. (1997). Effect Size: A Complete Sie of Statistical Significant, Egyptian Journal for Psychological Studies, 7 (16).

15. Farrell, K. E. (1999). Chaos Theory and Fractal Geometry: An Internet Curriculum, M.A. Thesis, California State University, (http://home.inreach.com/kfarrell.html

16. Gleick, J. (1987). Chaos, New York: Perguin Books.

17. Kellert, Stephen H. (1993). In the Wake of Chaos: Unpredictable Order in Dynamical Systems. Chicago: University of Chicago press.

18. Lornell, Randi; and Aesterberg, J. (1999). Fractals in High School: Exploring a New Geometry. The Mathematics Teacher, 92(3), March, PP. 260-265.

19. National Council of Teachers of Mathematics (2000). Principles and Standards for School Mathematics, Reston, $\mathrm{Va}, \mathrm{NCTM}$. 
20. O'Daffer, P. and Bruce, T. (1993). Critical Thinking, Mathematical Reasoning, and Proof. In Patricia, S. Ed, Research Ideas for the Classroom: High School Mathematics, New york, MacMillan Pub. Co.

21. Peitgen, H.; Jurgents, H.; Saupe, D.; Maletsky, E.; Pereiante, T.; and Yanker, L. (1992). Fractals for the Classroom: Introduction to Fractals and Chaos. Volume one, New York, Springer-Verlag.

22. Pressini, D. and Webb, N. (1999). Analyzing Mathematical Reasoning in Students Responses Across Multiple Performance Assessment Tasks' In Stiff, L. And Curcio, F. Editor; Developing Mathematical Reasoning in Grades k-12. Year Book, NCTM, Reston, VA

23. Royal Society (2001). Teaching and Learning Geometry 11-19: Report of a Royal Society/ Joint Mathematical Council working group. London: Royal Society.

24. Russell, S. J. (1999). Mathematical Reasoning in the Elementary Grades. In L. V. Stiff, \& F. R. Curcio (Eds.), Developing mathematical Reasoning in Grades K-12 (p. 1-12). Reston, VA: NCTM.

25. Simmt E.; and Daavis B. (1998). Fractal Cards: A space for Exploration in Geometry and Discrete Mathematics, The Mathematics Teacher, 91 (2), Feb., pp. 102-108.

26. Steen, L. A. (1997). Why numbers count: Quantative Literacy for Tomrrow's America. NY: The College Board.

27. Stewart, Ian(1998). The Mathematics of Chaos. Blackwell Publishers Inc., Ma.,

28. Van Hiele, P. (1986). Structure and Insight: a theory of mathematics education. Orlando FL, Academic Press.

29. Yi, T. (2004). A technology- Enhanced Fractal/Chaos Course. Electronic proceedings of the seventeenth annual international conference on technology in colligate mathematics, New Orleans, Oct. 28-31. http://archives. math.utk.edu/ICTCM/EP-17/C21/pdf/paper.pdf

\section{Appendix 1}

\section{Achievement Test: "Chaos Theory and Fractal Geometry"}

Solve the following questions; show all solving steps you follow:

1. Start with the equation $f(x)=0.1 x+0.4$. Begin the iteration with $x_{0}=0.4$ and use the output as the new input $x_{1}$. Continue repeating the process for successive values of $x$. Express as a fraction the limiting value of this iteration process.

2. What geometric properties would appear in a Spiro lateral generated from the number sequence 12345 on a square grid?

3. Iteration is performed through the function $\mathrm{f}(\mathrm{x})$ using the special relationship $x_{i+1}=f\left(x_{i}\right)$. Write equations that can be used through this iteration to generate the decimals for $\frac{1}{11}$ and $\frac{1}{7}$

4. Do the Koch snowflake iteration using squares instead of triangles. The first iteration is shown:

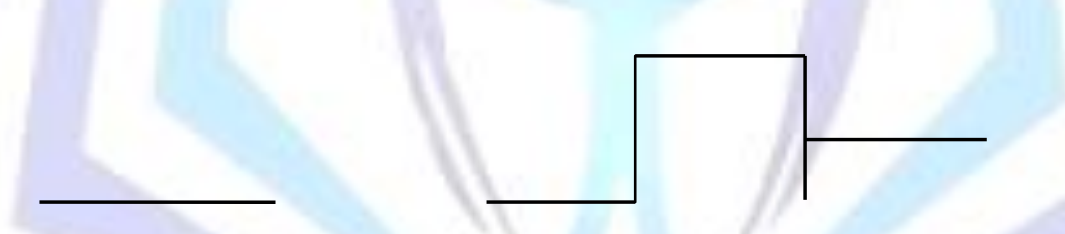

5. If the largest equilateral triangle in the diagram is 1 inch on each side and if the "spiral" shown is continued indefinitely, what will be the total length of the spiral?
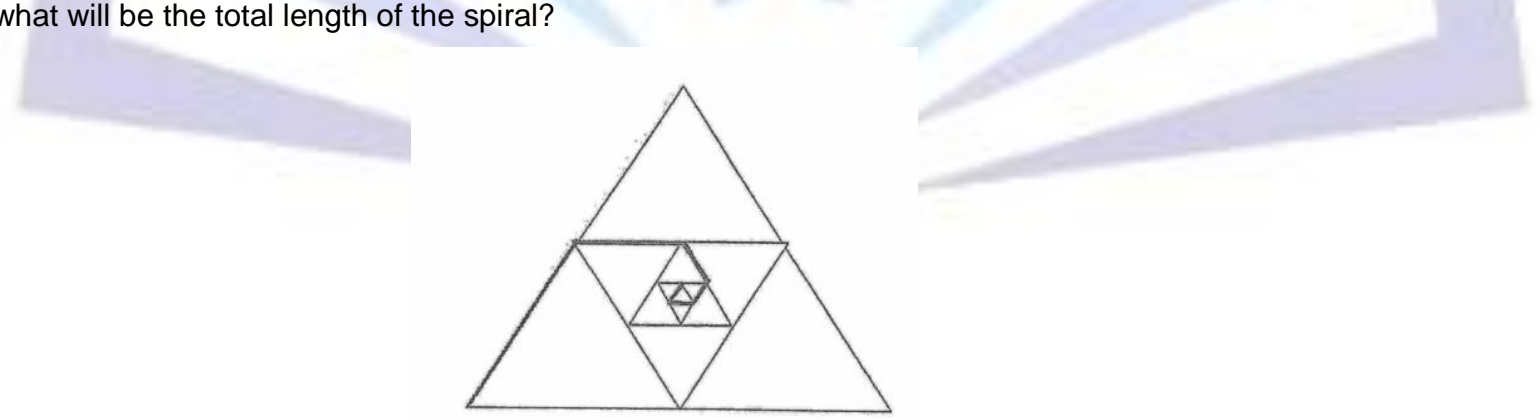

6. Starting with a square of side 1 unit, erase the middle third of each side, and replace it with a square going inwards. Do the same each side of the resulting figure, and keep repeating the process. Stages 2, 3 and 4 of this pattern are shown in figure. Find the length of the boundary and the area enclosed by stages 1, 2, 3, 4 and $n$. what can you say about the length and the area $n \rightarrow \infty$.

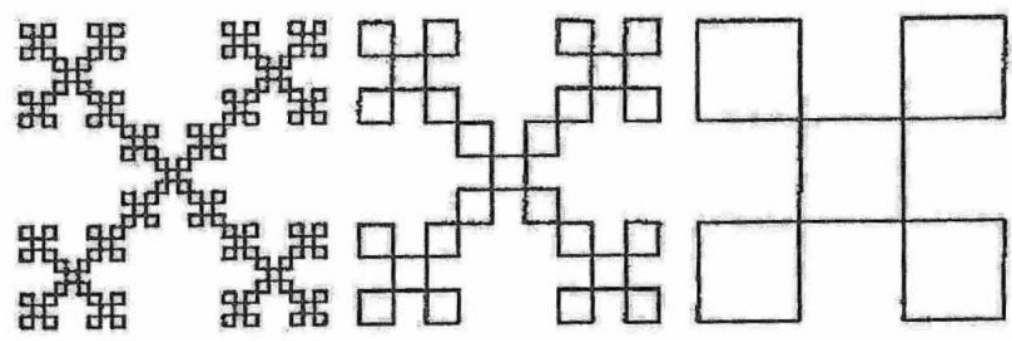




\section{Appendix 2}

\section{Geometric Reasoning Skills Test}

First: Answer the following questions

1. Write a new procedure called KOCHISLAND, which starts with a square (level1). Grow your KOCHISLAND by replacing each unit segment in the square with a zigzag path composed of eight smaller units each one-fourth as long as a side of the original square (level2).

Replace this:

with this:
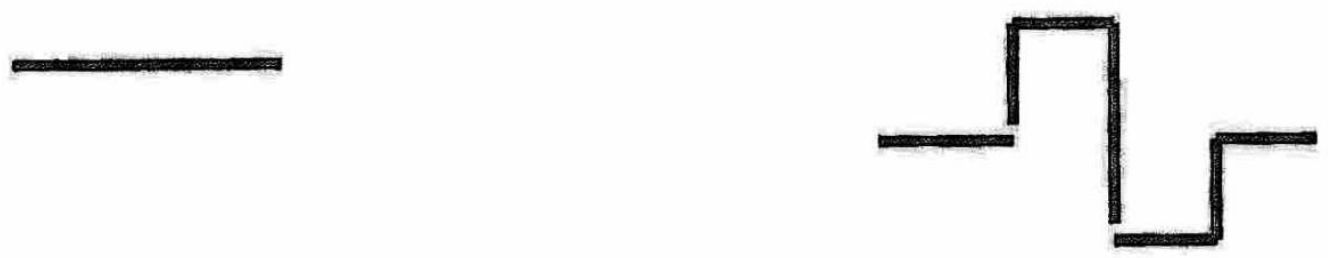

Level 1 (Koch Island)

Level 2 (Koch Island)

2. Study the pattern of the fractal octahedron below and complete the missing data on the table:

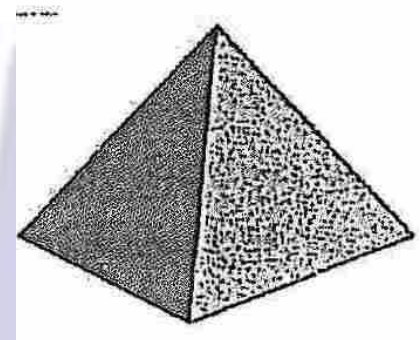

Stage 0

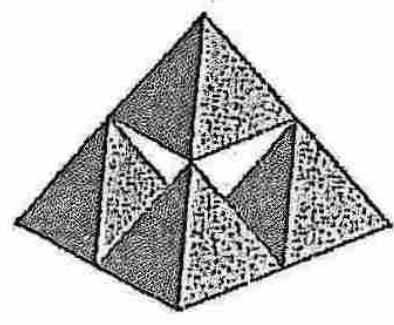

Stage 1

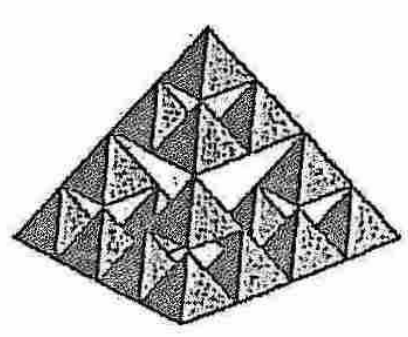

Stage 2

Table 3

\begin{tabular}{|llllllll|}
\hline Stage & 0 & 1 & 2 & 3 & 4 & n \\
\hline N. of Tetrahedrons & 1 & 4 & 16 & 64 & $?$ & $?$ \\
\hline Volumes & 1 & $\frac{1}{2}$ & $\frac{1}{4}$ & $\frac{1}{8}$ & $?$ & $?$ \\
\hline
\end{tabular}

3. If you start with a $4 \mathrm{~cm}$ square pice of paper. And you are folding the four corners. How do the area and perimeter change from one stage to the next? Complete the missing data in the table:

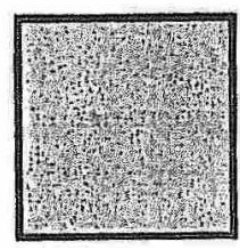

Stage 0

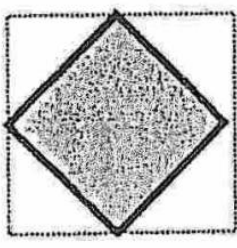

stage 1



Stage2

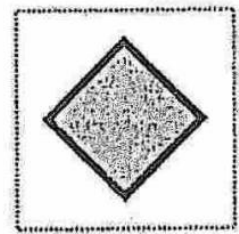

stage 3

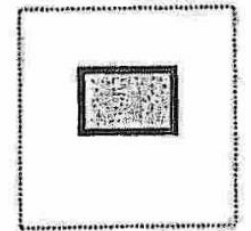

stage 4

Table 4

\begin{tabular}{|l|l|l|l|l|l|l|}
\hline Stage & 0 & 1 & 2 & 3 & 4 & $n$ \\
\hline Area & 16 & 8 & 4 & 2 & 1 & $?$ \\
\hline Perimeter & 16 & $?$ & 8 & $4 \sqrt{2}$ & 4 & $(\sqrt{2})^{?-n}$ \\
\hline
\end{tabular}


Second: Choose the right answer:

4. What is the next series of triangles in the sequence below?

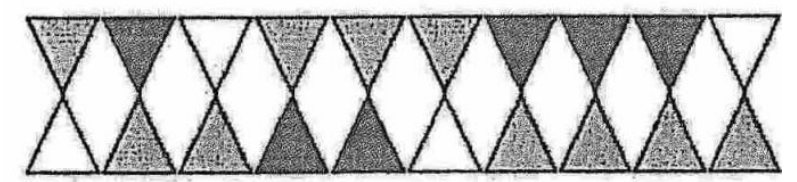

A.

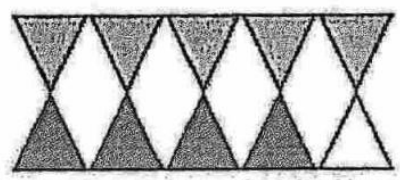

B.

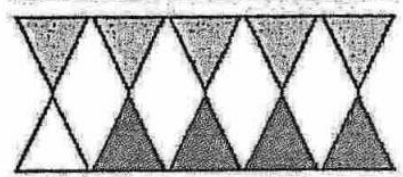

C.

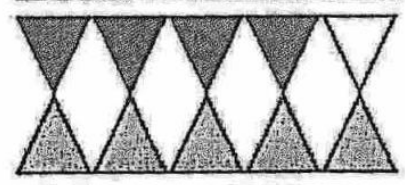

D.

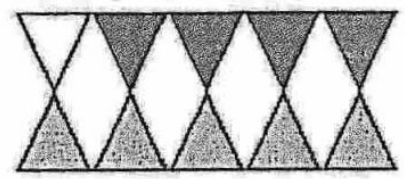

5. Write another form of the following statement. If a closed figure has exactly 3 vertices, then it is a triangle:

A. If a triangle has exactly 3 vertices, then it is a closed figure.

$B$. If a figure is closed, then it is a triangle with exactly 3 vertices

C. A closed figure is a triangle if it has exactly 3 vertices

D. A triangle is a closed figure if it has exactly 3 vertices

6. Write a converse for the statement, "If a figure has five angles, then it is a pentagon"

A. "A five angled figure is a pentagon"

B. "A pentagon is a figure with five angles"

C. "Pentagons have five angles and five sides"

D. "If a figure is a pentagon, then it has five angles"

7. How many different lines can be drawn using the points below?

\section{A *}

B*

D*
A. 8
B. 4
C. 6
D. 10 